\title{
Effect of vegetarian diets on the presentation of metabolic syndrome or its components: A systematic review and meta-analysis
}

\section{( 国 Article in press ? )}

- $\quad$ Picasso, M.C. ${ }^{a}$ Email Author,

- Lo-Tayraco, J.A. ${ }^{\mathrm{a}}$ Email Author,

- Ramos-Villanueva, J.M. ${ }^{a}$ Email Author,

- Pasupuleti, V. ${ }^{\mathrm{b} E m a i l}$ Author,

- Hernandez, A.V. ${ }^{\mathrm{c}, \mathrm{d}}$ Email Author

- View Correspondence (jump link)

- $\quad{ }^{\text {aS }}$ School of Nutrition, Faculty of Health Sciences, Universidad Peruana de Ciencias Aplicadas (UPC), Lima, Peru

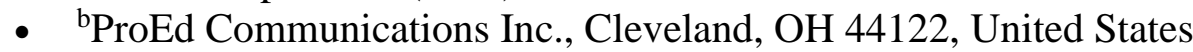

- ${ }^{\mathrm{c} S}$ School of Medicine, Universidad Peruana de Ciencias Aplicadas (UPC), Lima, Peru

$\underline{\text { View additional affiliations }}$

\begin{abstract}
Background \& aims: Several studies have examined the effect of vegetarian diets (VD) on metabolic syndrome (MetS) or its components, but findings have been inconsistent. The aim of this study was to perform a systematic review and meta-analysis of randomized controlled trials (RCTs) and observational studies to assess the association between VD and MetS or its components (systolic blood pressure [SBP], diastolic blood pressure [DBP], fasting glucose triglycerides, waist circumference [WC], HDLcholesterol (HDL-C)) in adults. Methods: The Cochrane Library, EMBASE, PubMed, Web of Science, and Scopus were searched. RCTs, cohort studies and cross-sectional studies evaluating the effects of VD on MetS or its components in adults, with omnivore diet as control group, were included. Random effects meta-analyses stratified by study design were employed to calculate pooled estimates. Results: A total of 71 studies ( $\mathrm{n}=$ 103008 ) met the inclusion criteria (6 RCTs, 2 cohorts, 63 cross-sectional). VD were not associated with MetS in comparison to omnivorous diet (OR 0.96, 95\% CI 0.50-1.85, p $=0.9$ ) according to meta-analysis of five cross-sectional studies. Likewise, metaanalysis of RCTs and cohort studies indicated that consumption of VD were not associated with MetS components. Meta-analysis of cross-sectional studies demonstrated that VD were significantly associated with lower levels of SBP (mean difference $[\mathrm{MD}]-4.18 \mathrm{mmHg}, 95 \% \mathrm{CI}-5.57$ to $-2.80, \mathrm{p}<0.00001)$, DBP (MD -3.03 $\mathrm{mmHg}, 95 \% \mathrm{CI}-4.93$ to $-1.13, \mathrm{p}=0.002)$, fasting glucose $(\mathrm{MD}-0.26 \mathrm{mmol} / \mathrm{L}, 95 \%$ $\mathrm{CI}-0.35$ to $-0.17, \mathrm{p}<0.00001)$, WC (MD $-1.63 \mathrm{~cm}, 95 \% \mathrm{CI}-3.13$ to $-0.13, \mathrm{p}=0.03)$, and HDL-C (MD $-0.05 \mathrm{mmol} / \mathrm{L}, 95 \% \mathrm{CI}-0.07$ to $-0.03, \mathrm{p}<0.0001)$ in comparison to omnivorous diet. Heterogeneity of effects among cross-sectional studies was high. About, one-half of the included studies had high risk of bias. Conclusions: VD in
\end{abstract}


comparison with omnivorous diet is not associated with a lower risk of MetS based on results of meta-analysis of cross-sectional studies. The association between VD and lower levels of SBP, DBP, HDL-C, and fasting glucose is uncertain due to high heterogeneity across the cross-sectional studies. Larger and controlled studies are needed to evaluate the association between VD and MetS and its components. (C) 2018 Elsevier Ltd and European Society for Clinical Nutrition and Metabolism

\section{Reaxys Database Information}

(1) View Compounds

\section{Author keywords}

Blood lipidsBlood pressureGlucoseMetabolic syndromeVegetarian dietsWaist circumference

- ISSN: 02615614

- CODEN: CLNUD

- Source Type: Journal

- Original language: English

- DOI: $10.1016 /$ j.clnu.2018.05.021

- Document Type: Article in Press

- Publisher: Churchill Livingstone 